\title{
AN EXACT INVERSE METHOD FOR SUBSONIC FLOWS*
}

\author{
BY \\ PRABIR DARIPA ${ }^{1,2,3}$ \\ Courant Institute of Mathematical Sciences, New York University
}

\begin{abstract}
A new inverse method for aerodynamic design of airfoils is presented for subcritical flows. The pressure distribution in this method can be prescribed in a natural way, i.e., as a function of arclength of the as yet unknown body. This inverse problem is shown to be mathematically equivalent to solving only one nonlinear boundary value problem subject to known Dirichlet data on the boundary. The solution to this nonlinear problem determines the airfoil, free stream Mach number $M_{\infty}$ and the upstream flow direction $\theta_{\infty}$. The existence of a solution to a given pressure distribution is discussed. The method is easy to implement and extremely efficient. We present a series of results for which comparisons are made with the known airfoils. This method will be extended to design supercritical airfoils in the future.
\end{abstract}

1. Introduction. The inverse problem in the context of aerodynamics is the determination of an airfoil that will generate a given pressure distribution. This problem has been considered important for decades because many desirable features of the flowfield, such as delay of separation and laminar-turbulent transition, can be achieved by proper prescription of the pressure distribution along the surface of the as yet unknown body (Stratford [33, 34, 35]). The point of separation of zero skin friction is related to the specified pressure distribution (see Stratford [33, 34] and references therein) and a flow with zero skin friction throughout its region of pressure rise is expected to achieve any specified pressure rise in the shortest possible distance with least possible energy loss (Stratford [35]). In aerodynamics these flow features are highly desirable and can be realized in practice since a suitable pressure distribution can be specified to avoid the undesirable flow features and an airfoil can be generated that will have this pressure distribution along its body. This paper addresses this problem of generating an airfoil from a given pressure distribution and proposes a new and extremely efficient method of solution to this problem.

\footnotetext{
${ }^{*}$ Received June 24, 1987.

${ }^{1}$ Supported in part by the Applied Mathematical Sciences subprogram of the Office of Energy Research, U. S. Department of Energy, under contract DE-AC02-76ER03077.

${ }^{2}$ Supported in part by the National Aeronautics and Space Administration under Grant NSG-1617.

${ }^{3}$ Present address: Department of Mathematics, Texas A \& M University.
} 
Various methods, iterative and noniterative, have evolved over the years to solve the inverse problem, mostly based on analytic function theory for incompressible flows (Arlinger [1], Glauert [13], Goldstein [14], Halsey [15], Johnson [21], Johnson and Rubbert [22], Langley [23], Lighthill [25], Strand [32]). However, for compressible flows, most of the solution techniques rely on solving a series of nonlinear boundary value problems in the physical or hodograph plane subject to either Dirichlet or Neumann boundary conditions, depending on the choice of the dependent variable. In either of these methods, an initial contour of the airfoil is guessed. In the Dirichlet formulation (Volpe [38], Volpe and Melnik [39], Tranen [37], Carlson [4]) a sequence of boundary value problems for the velocity potential, with wing geometry updated at each step, is solved. The updated condition arises from the normal velocity resulting at each unconverged step. In the Neumann formulation (Davis [8]; Hicks et al [19, 20]; McFadden [26]) a sequence of analysis problems is solved over a corresponding series of geometries. Each geometry is provided by some rational method depending on the difference between the computed speed and prescribed speed being driven to zero (for a review see Sloof [28]).

The above methods are very general and are applicable to subcritical as well as supercritical flows. The method of complex characteristics for solving the inverse problem in the case of supercritical flows is quite efficient and mathematically elegant (Bauer et al [2], Garabedian and Korn [11], Garabedian [10], Garabedian and McFadden [12], Sanz [27]). In their method the boundary is unknown and they iterate on the boundary to generate the airfoil. There are cost effective methods based on the fictitious gas concept to generate supercritical airfoils (Sobieczky [29], Yu [40]). An excellent review on the design of supercritical airfoils and wings can be found in Sobieczky and Seebass [31]. There are other methods of designing airfoils (Hassan et al [17, 18], Hassan [16]) which are not truly inverse methods since the input pressure distribution in these methods is not prescribed in a natural way, i.e., along the surface of the airfoil.

In this paper we present an inverse method where only one nonlinear boundary value problem needs to be solved. The method is computationally very efficient. Previously Daripa and Sirovich [7] have described an exact inverse method for subsonic flows within the tangent gas approximation. In this paper we discuss a method that applies to subsonic flows in general. The key idea of this method is to formulate the problem in the potential plane with the speed $q$ (normalized by the sonic speed) as the dependent variable. The body maps into a slit in the potential plane (see Fig. 1). An input pressure distribution $C_{p}(s)$ determines the slit, the speed $q$ on the slit and the speed at infinity, denoted by $q_{\infty}$. A solution of the governing equations subject to the known values of speed $q$ on the slit determines the airfoil and a speed at infinity, which we refer to as $q_{\infty}^{c}$. For a solution to exist, the $q_{\infty}$ determined directly from the input $C_{p}$ and the computed $q_{\infty}^{c}$ must be the same, or equivalently, $M_{\infty}$ must be the same as $M_{\infty}^{c}$. (The free stream Mach number is a function of the speed at infinity. $M_{\infty}$ and $M_{\infty}^{c}$ respectively refer to the free stream Mach number corresponding to $q_{\infty}$ and $q_{\infty}^{c}$.) Otherwise a solution with the input pressure distribution does not exist. In our method we always generate an airfoil, but the input and computed pressure 
distributions do not match unless the existence criterion, $q_{\infty}^{c}=q_{\infty}\left(\right.$ or $\left.M_{\infty}^{c}=M_{\infty}\right)$ is met. The input speed distribution and the calculated $M_{\infty}^{c}$ can be used to compute the pressure distribution over the designed airfoil at this Mach number $M_{\infty}^{c}$. The computed pressure distribution will be close to the input pressure distribution if $M_{\infty}$ is close to $M_{\infty}^{c}$.

The constraints due to the closure condition have not been imposed in this paper. Thus the designed airfoil could have a gap at the trailing edge. Currently under study is the inclusion of the closure condition as an exact constraint and the extension of the present method to design supercritical airfoils.

The paper is organized as follows. In Sec. 2 the governing equations of the steady, inviscid, irrotational flows in the potential plane are presented. These equations are then used to derive an equation in the potential plane with only one dependent variable, namely speed. In Sec. 3 the mapping of the potential plane to the interior of a unit circle is discussed. The governing equation in the plane of the circle as well as various relations that are relevant to our problem are derived in this section. Section 4 outlines the method of solution and presents the numerical results. Closing remarks are made in Sec. 5.

2. Basic equations. In this section we consider steady two-dimensional compressible fluid flow past an airfoil. We assume that the fluid flow is both irrotational and isentropic and that the fluid is a perfect gas obeying the gamma law equation of state. The governing equations of such a flow in usual notation are then given by

$$
\nabla \cdot(\rho \mathbf{q})=0 ; \quad \nabla \times \mathbf{q}=0 ; \quad p=\rho^{\gamma} .
$$

The variables are normalized with respect to their sonic values and linear dimensions with respect to some appropriate linear dimension. Here $p$ is the pressure, $\rho$ is the density, and $q$ is the speed. The equations $(1 \mathrm{a}, \mathrm{b})$ can be alternatively represented in terms of a potential $\phi$ and a stream function $\psi$, given by

$$
\rho \mathbf{q}=c \nabla \times(\psi \mathbf{k}) ; \quad \mathbf{q}=\nabla \phi,
$$

where $\mathbf{k}$ denotes a unit vector perpendicular to the plane of the motion. The constant $c$ has been introduced for later purposes.

If the potential function $\phi$ and the stream function $\psi$ are used as independent variables and the flow direction $\theta$ and the Prandtl-Meyer function $\nu$, defined by

$$
\nu=\int^{q}\left(\left|1-M^{2}\right|\right)^{1 / 2} \frac{d q}{q}
$$

are used as dependent variables, then the governing equations for the subcritical flows in the potential plane $w=(\phi+i \psi)$ are given by (Daripa and Sirovich [6], Bers [3])

$$
\theta_{\phi}-(K(M))^{-1} \nu_{\psi}=0 ; \quad \theta_{\psi}+(K(M)) \nu_{\phi}=0
$$

The function $K(M)$ in Eqs. (3a,b) is a function of the Mach number $M$ and is given by

$$
K(M)=\beta \frac{c}{\rho(q(M))}
$$


where

$$
\beta^{2}=\left|1-M^{2}\right| \text {. }
$$

The airfoil in the physical $(z=x+i y)$ and the potential $(w=\phi+i \psi)$ planes is shown in Fig. 1.

The system (3) should be solved subject to the ideal gas pressure-density relation (1c) and Bernoulli's relation given by

$$
\frac{q^{2}}{2}+\frac{1}{\gamma} \int \frac{d p}{\rho}=\text { constant. }
$$

The function $K(M)$ and the Prandtl-Meyer function $\nu(M)$ are related to the Mach number $M$ (Daripa [5]) as

$$
\begin{aligned}
& K(M)=c \beta\left(1-\left(\frac{\beta}{\kappa}\right)^{2}\right)^{\frac{1}{(\gamma-1)}}, \\
& \nu(M)=\kappa \tanh ^{-1}(\beta / \kappa)-\tanh ^{-1} \beta,
\end{aligned}
$$

where $\kappa$ is a function of the specific heat ratio, $\gamma$, given by

$$
\kappa^{2}=\frac{(\gamma+1)}{(\gamma-1)}
$$

Differentiation and elimination reduces the system $(3 a, b)$ of first-order partial differential equations (PDEs) to an equivalent second-order PDE in $\nu$ only, given by

$$
\left(K^{-1}(M) \nu_{\psi}\right)_{\psi}+\left(K(M) \nu_{\phi}\right)_{\phi}=0 \text {. }
$$

In the following paragraphs we briefly outline the underlying ideas, followed by a detailed account of our method.

It is straightforward to derive from (1c) and (6) the functional relationship $C_{p}=$ $C_{p}\left(M, M_{\infty}\right)$ and $q=q(M)$. See Daripa [5] and Liepmann and Roshko [24]. These relationships determine the free stream Mach number $M_{\infty}$, the Mach number $M(s)$, and speed $q(s)$ along the surface of the body from any specified pressure distribution $C_{p}(s), 0 \leq s \leq 1$, where $s=0$ and 1 respectively refer to the upper and lower side of the trailing edge of the airfoil. A correspondence between the body surfaces in the physical and potential planes is made through the relation $|d \phi|=q(s) d s$ which determines $\phi(s)$ upon integration. A knowledge of $M(s)$ and $\phi(s)$ together determines $M(\phi)$ and hence $\nu(M(\phi))$ on the slit in the potential plane (see Fig. 1). The solution of Eq. (10) subject to the known values of $\nu(\phi)$ then determines $\nu_{\psi}$ on the slit. The normal derivative $\nu_{\psi}$ on the slit can be used in Eq. (3a) to find the value of $\theta_{\phi}$ and hence the body.

The solution of the elliptic equation (10) subject to the Dirichlet data on the slit also determines the free stream Mach number $M_{\infty}^{c}$. The superscript $c$ refers to the computed value. The free stream Mach number $M_{\infty}$ associated with the input pressure distribution $C_{p}(s)$ need not be the same as the computed value $M_{\infty}^{c}$. $M_{\infty}=M_{\infty}^{c}$ will imply existence of a solution (an airfoil) corresponding to the input pressure distribution. (This is equivalent to the condition due to the mean value theorem in the linear case (Laplace equation).) If $M_{\infty} \neq M_{\infty}^{c}$, then there does not exist a solution corresponding to the given pressure distribution $C_{p}(s)$. However, 


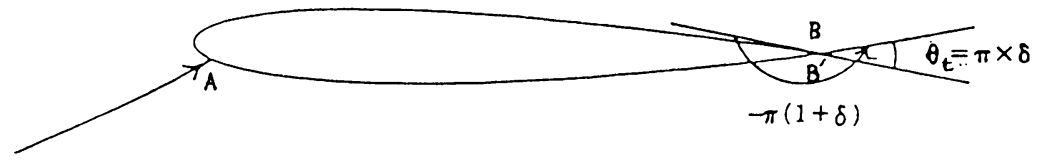

$$
\text { z-plane }
$$

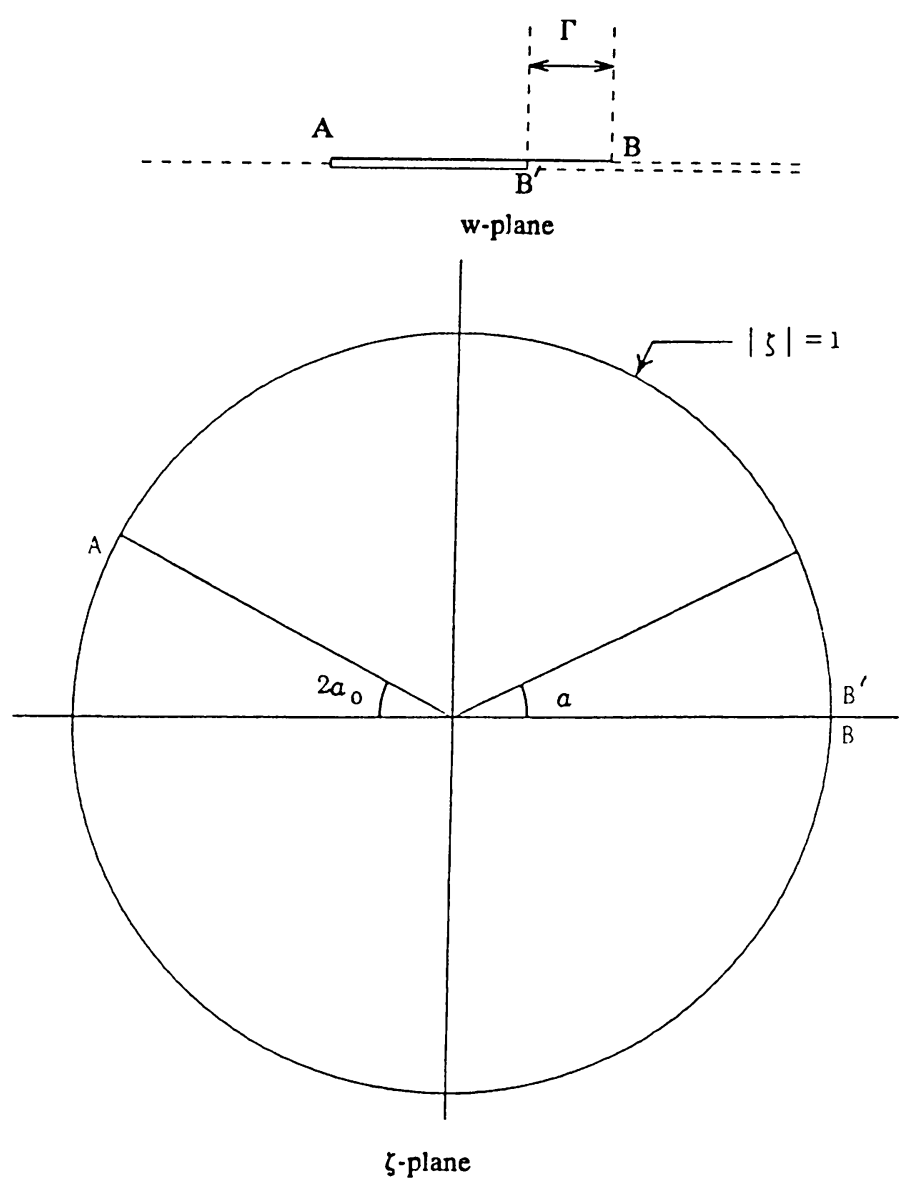

Fig. 1. Airfoil in the physical $z$-plane, potential $w$-plane and the circle $\zeta$-plane. A is the front stagnation point.

notice that in our formulation we always generate a body. If $M_{\infty}^{c}$ is different from $M_{\infty}$, then the pressure distribution on the designed body can be calculated easily at $M_{\infty}^{c}$, using this value for $M_{\infty}$ in the formula for $C_{p}$. However, one can also solve the analysis problem directly on the designed airfoil to compute the pressure distribution.

Details of our method follow. The governing equation (10) can alternatively be written as

$$
\nabla_{\phi, \psi}^{2} \nu=\left(1-K^{2}\right) \nu_{\phi \phi}+K^{-1} K_{\psi} \nu_{\psi}-K K_{\phi} \nu_{\phi}
$$

Since $K$ and $\nu$ are functions of Mach number only, it follows that

$$
K_{\psi}=K_{M} M_{\nu} \nu_{\psi}, \quad K_{\phi}=K_{M} M_{\nu} \nu_{\phi}
$$


Equations $(12 a, b)$ reduce Eq. (11) to

$$
\nabla_{\phi, \psi}^{2} \nu=\left(1-K^{2}\right) \nu_{\phi \phi}+f(M) \nu_{\psi}^{2}-K^{2} f(M) \nu_{\phi}^{2}
$$

where $f(M)$ is given by (Daripa [5])

$$
f(M)=K_{M} \frac{M_{\nu}}{K}=-\frac{\gamma+1}{2} M^{4}\left(\left|1-M^{2}\right|\right)^{-3 / 2} .
$$

Use of Eq. (7) has been made to derive (14). Equation (13) is the governing equation for $\nu$ and Eqs. (3a,b) for the flow direction $\theta$. It will be useful to transform the equations from an infinite domain of the $w$-plane into a finite domain of some new plane. This is discussed in the next section.

\section{Equations in the circle plane.}

3.1. Mapping. The potential plane $w$ is mapped into the interior of the unit circle in the plane $\zeta=r e^{i \alpha}$ such that the body maps onto the unit circle and the infinity goes into the origin. This mapping function is given by (Daripa and Sirovich [6, 7])

$$
w=a\left(\zeta e^{i \alpha_{0}}+\zeta^{-1} e^{-i \alpha_{0}}\right)-i 2 a \sin \alpha_{0} \ln \left(\zeta e^{i \alpha_{0}}\right) .
$$

Here the constants $a$ and $\alpha_{0}$, as yet unknowns, relate to two physical variables, namely the free stream direction with respect to the as yet unknown body and the circulation. As seen from this mapping the circulation, $-\Gamma$, is related to the constants $a$ and $\alpha_{0}$ by

$$
\Gamma=4 \pi a \sin \alpha_{0} .
$$

The relation of the free stream flow direction, $\theta_{\infty}$, to the body is discussed in Sec. 3.6. From (15) we see

$$
\frac{d w}{d \zeta}=-a e^{-i \alpha_{0}}\left(1-\zeta^{-1}\right)\left(e^{-i \alpha_{s}}-\zeta^{-1}\right)
$$

where

$$
\alpha_{s}=\pi-2 \alpha_{0}
$$

Thus the rear and front stagnation points map into $\zeta=1$ and $\zeta=e^{i \alpha_{s}}$ respectively. Notice that the lower side of the trailing edge maps into $\alpha=0$ and the upper side into $\alpha=2 \pi$ on the unit circle. On the body $\zeta=e^{i \alpha} ; 0 \leq \alpha \leq 2 \pi, \phi$ and $\psi$ are given by

$$
\phi(\alpha)=2 a \cos \left(\alpha+\alpha_{0}\right)+2 a\left(\alpha+\alpha_{0}\right) \sin \alpha_{0} ; \quad \psi(\alpha)=0 .
$$

Next we discuss the determination of the speed on the unit circle from an input pressure distribution $C_{p}(s)$.

3.2. Speed distribution on the unit circle. The following relations can be derived using (1c) and (6) through simple algebraic manipulation. See Daripa [5] and Liepmann and Roshko [24].

$$
C_{p}=\frac{2}{\gamma M_{\infty}^{2}}\left(\frac{p}{p_{\infty}}-1\right)
$$

where the pressure $p$ is given by

$$
\frac{1}{p}=\left(1-\frac{\beta^{2}}{\kappa^{2}}\right)^{\frac{\gamma}{\gamma-1}}
$$


$p_{\infty}$ in (20) refers to the value of $p$ at infinity. The speed $q$ and the Mach number $M$ are related to the pressure $p$ through

$$
q^{2}=\kappa^{2}-\left(\kappa^{2}-1\right) p^{(\gamma-1) / \gamma}, \quad M=1-\kappa^{2}\left(1-p^{(1-\gamma) / \gamma}\right) .
$$

Relations (20) through (22) determine $M(s), q(s)$, and $M_{\infty}$ from given $C_{p}(s)$. Since the body is a streamline, the following relation follows from $(2 b)$ :

$$
q_{s} d s=|d \phi| .
$$

$s=0$ denotes the lower side of the trailing edge and $s=s_{f}$ denotes the distance of the front stagnation point from the lower side of the trailing edge. Equation (19) reduces Eq. (23) to

$$
q_{s} d s=2 a\left|\sin \alpha_{0}-\sin \left(\alpha+\alpha_{0}\right)\right| d \alpha ; \quad 0 \leq \alpha \leq 2 \pi,
$$

which is simply an ordinary differential equation for $s(\alpha)$. To integrate (24) we introduce

$$
d Q=d \int_{0}^{s} q_{s}\left(s^{\prime}\right) d s^{\prime}
$$

from which

$$
\begin{aligned}
Q(\alpha) & =Q(s(\alpha)) \\
& = \begin{cases}2 a\left\{\left(\cos \alpha_{0}-\alpha \sin \alpha_{0}-\cos \left(\alpha+\alpha_{0}\right)\right\},\right. & 0 \leq \alpha \leq \alpha_{s} \\
2 a\left\{2\left(\cos \left(\alpha-\alpha_{s} \sin \alpha_{0}\right)+\left(\alpha \sin \alpha_{0}+\cos \left(\alpha+\alpha_{0}\right)+\cos \alpha_{0}\right)\right\},\right. & \alpha_{s} \leq \alpha \leq 2 \pi .\end{cases}
\end{aligned}
$$

Observe that

$$
\begin{gathered}
Q(s=1)=8 a\left(\alpha_{0} \sin \alpha_{0}+\cos \alpha_{0}\right), \\
Q(s=1)-Q\left(s=s_{f}\right)=2 a\left\{\cos \alpha_{0}+\left(\pi+2 \alpha_{0}\right) \sin \alpha_{0}\right\} .
\end{gathered}
$$

$\Gamma$ is related to $Q(s=1)$ and $Q\left(s=s_{f}\right)$ by

$$
\Gamma=2 Q(s=1)-Q\left(s=s_{f}\right)=4 \pi a \sin \alpha_{0} .
$$

$Q(s=1), Q\left(s=s_{f}\right)$, and $\Gamma$ are known from $q_{s}(s)$ (see Eqs. (25) and (28)). From $(27 a)$ and $(28)$ it follows

$$
\frac{Q(s=1)}{\Gamma}=\frac{2}{\pi}\left(\alpha_{0}+\cot \alpha_{0}\right) .
$$

After (29) is solved for $\alpha_{0}$, the constant $a$ can be calculated from (28). Next $Q(\alpha)$ is computed from (26) and $s(\alpha)$ is obtained by inverting (26),

$$
s(\alpha)=Q^{-1}(Q(\alpha)) \text {, }
$$

and $\tilde{q}_{s}(\alpha)=q_{s}(s(\alpha))$ is obtained from (24). This determines the speed and hence the Mach number $M$ (see Eq. (22b)) and the Prandtl-Meyer function $\nu$ (see Eq. (8)) on the unit circle.

As it will be seen, the airfoil and the flow direction at infinity are related to the values of $\nu$ inside the unit circle. This requires solving the governing equation (13) in the circle plane subject to the known values of $\nu$ on the unit circle. Next we discuss this equation in the circle plane and the relation of the airfoil and the flow direction at infinity to the values of $\nu$ inside the unit circle. 
3.3. Transformed equations. The conformal mapping (15) transforms Eq. (13) into

$$
\nabla_{r, \alpha}^{2} \nu=\frac{D}{r^{2}}\left[\left(1-K^{2}\right) \nu_{\phi \phi}+f(M) \nu_{\psi}^{2}-K^{2} f(M) \nu_{\phi}^{2}\right]
$$

where

$$
\nabla_{r, \alpha}^{2} \nu=\frac{1}{r} \partial_{r}\left(r \partial_{r} \nu\right)+\frac{1}{r^{2}} \partial_{\alpha \alpha} \nu,
$$

and $\nu_{\phi \phi}, \nu_{\psi}, \nu_{\phi}$, and $D$ are known functions of the various derivatives of $\phi, \psi$, and $\nu$ in the circle plane and can easily be derived using the mapping function (15).

The Prandtl-Meyer function $\nu$ (see Eq. (8)) has a logarithmic singularity in speed $q$ at a stagnation point. It is essential to remove this singularity before the solution of Eq. (31) is sought. This is discussed next.

3.3.1. Removal of singularity. The logarithmic singularity of $\nu$ at a stagnation point is removed by introducing

$$
\widetilde{\nu}(r, \alpha)=-\nu(r, \alpha)+L(r, \alpha)
$$

where

$$
L(r, \alpha)=-\ln G(r, \alpha) .
$$

$G(r, \alpha)$ in Eq. (34) is given by

$$
G(r, \alpha)=\left|(1-\zeta)^{-\delta}\left(e^{i \alpha_{s}}-\zeta\right)^{-1}\right|=\left(S_{1}(r, \alpha)\right)^{-\delta / 2}\left(S_{2}(r, \alpha)\right)^{-1 / 2}
$$

where

$$
S_{1}(r, \alpha)=1+r^{2}-2 r \cos \alpha ; \quad S_{2}(r, \alpha)=S_{1}\left(r, \alpha-\alpha_{s}\right) .
$$

$\delta$ denotes $\theta_{t} / \pi$ where $\theta_{t}$ is the trailing edge angle. Then Eq. (31) becomes

$$
\nabla_{r, \alpha}^{2} \widetilde{\nu}=\frac{D}{r^{2}}\left[\left(1-K^{2}\right)\left(\widetilde{\nu}_{\phi \phi}-L_{\phi \phi}\right)+f(M)\left(\widetilde{\nu}_{\psi}-L_{\psi}\right)^{2}-K^{2} f(M)\left(\widetilde{\nu}_{\phi}-L_{\phi}\right)^{2}\right] .
$$

Equation (37) is the main equation to be solved in the circle plane. The values of $\widetilde{\nu}(r=1, \alpha)$ are computed from known values of $\nu(r=1, \alpha)$ using Eq. (33). As we see from (34) to (36), $L(r, \alpha)$ is singular at the stagnation points $\alpha=0$ and $\alpha=\alpha_{s}$ on the unit circle and this singularity precisely cancels the singularity in $\nu$ at those points. Therefore, it is not straightforward to calculate the values of $\widetilde{\nu}$ using (33) at these stagnation points and special estimates have to be made to evaluate them at $\alpha=0$ and $\alpha=\alpha_{s}$. This will be discussed in Sec. 3.6. Various derivatives of $L$ and $\nu$ with respect to $\phi$ and $\psi$ in Eq. (37) can easily be related to their derivatives in the circle plane through the mapping function (15).

We have regularized $\nu$ but the right-hand side (henceforth to be referred to as RHS) of Eq. (37) remains singular at a stagnation point (see Appendix B). As shown in Appendix B, the singularity at a front stagnation point can be removed by choosing a particular value of the constant $c$ appearing in $K$ (see Eq. (4)) which is given by

$$
c=\left(\frac{\gamma+1}{2}\right)^{\frac{1}{\gamma-1}} \text {. }
$$

However, the RHS remains singular at the trailing edge $(\alpha=0)$ if $0<\theta_{t}<\pi / 2$. For the case where the trailing edge angle is zero, the RHS is regular also at the trailing 
edge and hence the RHS remains regular everywhere. We will only be concerned with this case when we discuss our results.

The solution of (37) subject to the known Dirichlet data will provide the value of $\widetilde{\nu}$ inside the unit circle. Next we discuss how to relate these values of $\widetilde{\nu}$ to the body angle $\theta_{B}(\alpha)$ and the free stream direction $\theta_{\infty}$.

3.4. Body angle. It is easy to see from (33) to (36) that on the unit circle

$$
\nu_{r}=-\widetilde{\nu}_{r}+\varepsilon+\text { constant } \times \widehat{\delta}\left(\alpha-\alpha_{s}\right)+\text { constant } \widehat{\delta}(\alpha) ; \quad \text { on } r=1,
$$

where

$$
\varepsilon=\frac{(1+\delta)}{2}
$$

and $\widehat{\delta}$ denotes the Dirac delta function. The tangent angle $\theta_{B}$ on the body is related to the flow direction $\theta$ on the body by

$$
\theta(\alpha)=\theta_{B}(\alpha)-2 \pi+\pi U\left(\alpha-\alpha_{s}\right)
$$

where $U\left(\alpha-\alpha_{S}\right)$ is the unit step function. Then

$$
\theta_{\alpha}(\alpha)=\left(\theta_{B}(\alpha)\right)_{\alpha}+\pi \widehat{\delta}\left(\alpha-\alpha_{s}\right)
$$

As shown in Appendix A (see equation (A6)), under the mapping (13), $\theta_{\alpha}$ on the unit circle is related to $\nu$ through the following relation:

$$
\theta_{\alpha}=-\left.K^{-1} \nu_{r}(\alpha)\right|_{r=1} .
$$

Direct substitution of (38) and (41) in (42) results in

$$
\left(\theta_{B}(\alpha)\right)_{\alpha}=K^{-1}\left(\left.\widetilde{\nu}_{r}\right|_{r=1}-\varepsilon\right) .
$$

From (43) it follows that

$$
\int_{0}^{2 \pi} K^{-1}\left(\left.\widetilde{\nu}\right|_{r=1}-\varepsilon\right) d \alpha=-2 \varepsilon \pi
$$

since (see Fig. 1)

$$
\theta_{B}(\alpha=2 \pi)-\theta_{B}(\alpha=0)=-2 \varepsilon \pi
$$

If $\widetilde{\nu}_{r}$ is known on the unit circle, then $\left(\theta_{B}(\alpha)\right)_{\alpha}$ is known and $\theta_{B}(\alpha)$ on the body can easily be computed by integration as given by

$$
\theta_{B}(\alpha)=\theta_{B}(\alpha=0)+\int_{0}^{\alpha} K^{-1}\left(\left.\widetilde{\nu}\right|_{r=1}-\varepsilon\right) d \alpha .
$$

$\theta_{B}(\alpha=0)$ can be arbitrarily assigned to calculate $\theta_{B}(\alpha)$. Then the body can easily be computed using the equations

$$
\begin{aligned}
& x(\alpha)=\int_{0}^{\alpha} \frac{d s}{d \alpha} \cos \theta_{B}\left(\alpha^{\prime}\right) d \alpha^{\prime}, \\
& y(\alpha)=\int_{0}^{\alpha} \frac{d s}{d \alpha^{\prime}} \sin \theta_{B}\left(\alpha^{\prime}\right) d \alpha^{\prime}
\end{aligned}
$$

with

$$
\frac{d s}{d \alpha}=2 a \frac{\left|\sin \alpha_{0}-\sin \left(\alpha+\alpha_{0}\right)\right|}{q_{s}(\alpha)} .
$$


The free stream direction $\theta_{\infty}$ must be related to $\theta_{B}(\alpha=0)$ to find the orientation of the body with respect to the free stream direction.

3.5. Free stream direction. $\theta_{r}$ can be integrated along any ray, $\alpha=$ constant, in the unit circle from the origin to the unit circle to calculate the free stream direction $\theta_{\infty}=\theta(r=0)$ with respect to the body. It is easy to see that

$$
\theta\left(r=1, \alpha=\alpha_{*}\right)=\theta_{\infty}+\left.\int_{0}^{1} \theta_{r}(r)\right|_{\alpha=\alpha_{*}} d r
$$

where $\alpha_{*}$ is the angular position of any ray. From (40) and (49)

$$
\theta_{B}\left(\alpha=\alpha_{*}\right)=\theta_{\infty}+\left.\int_{0}^{1} \theta_{r}(r)\right|_{\alpha=\alpha_{*}} d r+2 \pi-\pi U\left(\alpha-\alpha_{s}\right) .
$$

$\theta_{r}$ is related to $\nu_{r}$ and $\nu_{\alpha}$ in general through the relation (3a,b) and mapping function (15). Use of (33) and (A10) (see Appendix A) gives

$$
\theta_{r}=\left.\frac{K(M)}{r}\left(-\widetilde{\nu}_{\alpha}+L_{\alpha}\right)\right|_{\alpha=\alpha_{1}} ; \quad \alpha_{1}=\frac{\pi}{2}-\alpha_{0} .
$$

From Eqs. (49), (50), and (51) we see that $\theta_{B}(\alpha=0)$ is related to $\theta_{\infty}$ by

$$
\begin{aligned}
\theta_{B}(\alpha=0) & =\theta_{B}\left(\alpha=\alpha_{1}\right)-\int_{0}^{\alpha_{1}} K^{-1}\left(\left.\widetilde{\nu}_{r}\right|_{r=1}-\varepsilon\right) d \alpha \\
& =\theta_{\infty}+2 \pi-\int_{0}^{\alpha_{1}} K^{-1}\left(\left.\widetilde{\nu}_{r}\right|_{r=1}-\varepsilon\right) d \alpha+\left.\int_{0}^{1} \frac{K(M)}{r}\left(-\widetilde{\nu}_{\alpha}+L_{\alpha}\right)\right|_{\alpha=\alpha_{1}} d r .
\end{aligned}
$$

Equation (52) determines $\theta_{\infty}$ and hence the orientation of the body with respect to the free stream direction. So far our exposition has been exact. As mentioned in Sec. 3.3.1, we need to estimate the values of $\widetilde{\nu}(\alpha=0)$ and $\widetilde{\nu}\left(\alpha=\alpha_{s}\right)$ on the unit circle. This is discussed next, which will be useful later for numerical solution of (37). In the next section we also discuss how to compute the trailing edge angle $\delta\left(=\frac{\theta_{t}}{\pi}\right)$ from known values of speed on the unit circle.

3.6. Behavior at stagnation points. It is both interesting and useful to study the behavior near a stagnation point $\left(\zeta=0, \zeta=e^{i \alpha_{s}}\right)$. From (20) one obtains

$$
q \approx \frac{\kappa M}{\sqrt{\left(\kappa^{2}-1\right)}} ; \text { for } q \approx 0 .
$$

From (8) one obtains

$$
\tanh ^{-1} \beta \approx \kappa \tanh ^{-1}\left(\frac{1}{\kappa}\right)-\nu ; \text { for } q \approx 0
$$

From (5) and (54) one obtains

$$
M \approx 2\left\{\frac{(\kappa+1)}{(\kappa-1)}\right\}^{-\kappa / 2} e^{\nu} .
$$

From (53) and (55) it follows

$$
q \approx F(\kappa) e^{\nu} ; \quad \text { for } q \approx 0
$$

where

$$
F(\kappa)=2\left\{\frac{(\kappa+1)}{(\kappa-1)}\right\}^{-\kappa / 2} \frac{\kappa}{\sqrt{\left(\kappa^{2}-1\right)}}
$$


From (34), (35), and (56)

$$
\tilde{q} \approx F(\kappa) \frac{e^{-\tilde{\nu}}}{G(\alpha)} ; \text { for } q \approx 0 .
$$

A tilde on $q$ indicates it is a function of $\alpha$. From (36) and (37)

where

$$
G^{-1}(\alpha) \approx \begin{cases}\alpha^{\delta}\left(2 \cos \alpha_{0}\right) & \text { for } \alpha \approx 0 \\ \left|\alpha-\alpha_{s}\right|\left(2 \cos \alpha_{0}\right)^{\delta} & \text { for } \alpha \approx \alpha_{s}\end{cases}
$$

From (58) and (59)

$$
\tilde{q} \approx \begin{cases}K_{1} \alpha^{\delta} & \text { for } \alpha \approx 0, \\ K_{2}\left|\alpha-\alpha_{s}\right| & \text { for } \alpha \approx \alpha_{s},\end{cases}
$$

$$
\begin{aligned}
& K_{1}=F(\kappa) e^{-\tilde{\nu}(\alpha=0)}\left(2 \cos \alpha_{0}\right), \\
& K_{2}=F(\kappa) e^{-\widetilde{\nu}\left(\alpha=\alpha_{s}\right)}\left(2 \cos \alpha_{0}\right)^{\delta} .
\end{aligned}
$$

If $\alpha_{1}$ and $\alpha_{2}$ are close to $\alpha=0$, then from (60)

$$
\delta \approx \frac{\ln \left(\tilde{q}\left(\alpha_{2}\right) / \widetilde{q}\left(\alpha_{1}\right)\right)}{\ln \left(\alpha_{2} / \alpha_{1}\right)} .
$$

From (60) and (61)

$$
\widetilde{\nu}(\alpha=0) \approx-\ln \left[\frac{\widetilde{q}\left(\alpha_{1}\right)\left(2 \cos \alpha_{0}\right)^{-1}}{\alpha_{1}^{\delta} F(\kappa)}\right]
$$

and

$$
\widetilde{\nu}\left(\alpha=\alpha_{s}\right) \approx-\ln \left[\frac{\widetilde{q}\left(\alpha_{f}\right)\left(2 \cos \alpha_{0}\right)^{-\delta}}{\left|\alpha_{f}-\alpha_{s}\right| F(\kappa)}\right]
$$

where $\alpha_{f} \approx \alpha_{s}$. Equations (62) to (64) determine $\delta, \widetilde{\nu}(\alpha=0)$, and $\widetilde{\nu}\left(\alpha=\alpha_{s}\right)$ from $\widetilde{q}(\alpha)$. Having discussed all the relevant details, the method of solution is discussed next.

4. Numerical results. In this section we discuss the numerical steps involved in carrying out this design method. We present numerical results for two different (symmetric and nonsymmetric) pressure distributions corresponding to known airfoils.

4.1. Methods of solution. Usually, the pressure distribution $C_{p}(s), 0 \leq s \leq 1$, is prescribed at a finite number of points $s_{j}, j=1,2,3, \ldots, n$. We assume that $C_{p}\left(s=s_{f}\right)=C_{p, O}$ is one of the data points (this is the maximum value of $C_{p}(s)$ ) and corresponds to the stagnation point pressure coefficient. The free stream Mach number $M_{\infty}$ is obtained from $C_{p, O}$ using Eqs. (20) and (21). The calculation procedure goes as follows.

(a) The pressure $p_{s}(s)$, the Mach number $M(s)$, and the speed $q_{s}(s)$ on the body are computed from $C_{p}(s)$ using the relations (20) to (22). In all our cases the $C_{p}(s)$ was prescribed at 129 points.

(b) The speed $\widetilde{q}(\alpha)$ on the unit circle at $N$ grid points is computed from $q_{s}(s)$ (see Sec. 3.2). In all our runs the number of grid points on the unit circle in the mapped plane was 129. 
(c) The Mach number $M(\alpha)$ and the Prandtl-Meyer function $\nu(\alpha)$ are computed from $\widetilde{q}(\alpha)$ using the relations $(8)$ and $(22)$.

(d) The regularized Prandtl-Meyer function $\widetilde{\nu}(\alpha)$ is then computed using Eqs. (33) to (36) except at stagnation points $\left(\alpha=0\right.$ and $\left.\alpha=\alpha_{s}\right)$. Stagnation point estimates of $\widetilde{\nu}$ are obtained from (63) and (64) and the trailing edge angle $\delta$ from (62). Accuracy of these estimates depends on the accuracy in the speed $\widetilde{q}(\alpha)$ near these points (see Eqs. (63) and (64)) and hence on the speed distribution $q_{s}(s)$ near the stagnation points where the slope is usually steep. If the speed is not prescribed over a sufficient number of points in these steep areas, then the above estimate could be inaccurate. Under such circumstances, the above estimates of $\widetilde{\nu}(\alpha)$ should be obtained by interpolation and extrapolation near front and rear stagnation points, respectively, and the trailing edge angle should be prescribed.

In all our runs the approximate relations (63) and (64) were used to compute the stagnation point estimates. In addition, the input speed at the trailing edge was finite but not zero, which means that the trailing edge angle is zero.

(e) Equation (37) is solved numerically inside the unit circle subject to the following known data:

$$
\begin{gathered}
\widetilde{\nu}(r=1, \alpha)=\widetilde{\nu}(\alpha), \quad 0 \leq \alpha \leq 2 \pi, \\
\nabla_{r, \alpha}^{2} \widetilde{\nu}=0 \quad \text { at } r=0 .
\end{gathered}
$$

Notice that the second condition above follows since the flowfield is uniform at infinity. In solving Eq. (37) numerically, a general linear Helmholtz equation solver (Swarztrauber and Sweet [36]) is used in an iterative manner. An initial guess of the flowfield inside the unit circle determines the right-hand side (RHS) of Eq. (37) at $(M-2) \times N$ grid points where $M$ and $N$ are the number of uniformly spaced grid points in the radial and angular directions, respectively. The solver then updates the values of $\widetilde{\nu}$ inside the unit circle and the process is repeated until a given convergence criterion is met.

In all our runs $M \times N$ was taken to be $50 \times 129$. The solution was considered to have converged if the difference in the maximum value of the regular PrandtlMeyer function $\widetilde{\nu}$ inside the unit circle between two successive iterations was less than $5 \times 10^{-6}$. We never needed more than six iterations in all of our calculations when the initial guess was taken to be that of a uniform flowfield.

(f) After the solution $\widetilde{\nu}$ inside the unit circle is obtained in the above step, $\widetilde{\nu}_{r}\left(\alpha_{j}, r=\right.$ 1) for $j=1, \ldots, N$ and $\widetilde{\nu}_{\alpha}\left(r_{i}, \alpha=\pi / 2-\alpha_{0}\right)$ for $i=2, \ldots, M$, are computed using second-order finite difference equations.

(g) Equation (52) is used to find $\theta_{B}(\alpha=0)$ in relation to the free stream direction. Equation (46) then determines the body angle $\theta_{B}(\alpha)$ and the body is constructed using Eqs. (47) and (48). The trapezoid rule was used for numerically evaluating the integrals in Eqs. (46), (47), and (52).

(h) The solution of the elliptic equation (37) also determines $\widetilde{\nu}(r=0)$ and hence the Mach number $M(r=0)$ (see Eq. (8)). Since the origin of the circle plane corresponds to infinity in the physical plane (see Eq. (15)), the computed free stream Mach number $M_{\infty}^{c}=M(r=0)$. 
Even though the exact solution of Eq. (37) will give the exact airfoil, errors are introduced in the numerical approximations. The major source of error is in step (f). This could be improved by using higher-order finite difference approximations for $\widetilde{\nu}_{r}$.

4.2. Results. In this section we present a series of results using our method. The program was run on IBM 3081 in single precision and the computation time was approximately 5 minutes in the cases discussed below. Here we generate pressure distributions over a series of closed airfoils at a given free stream Mach number $M_{\infty}$ and angle of attack $\theta_{\infty}$ by using an Euler code (flo52s written by A. Jameson, E. Turkel, and M. Salas). This pressure distribution is then used in our method to generate the airfoil and to find the computed free stream Mach number, $M_{\infty}^{c}$, and the computed angle of attack, $\theta_{\infty}^{c}$. The diagnostics that we monitor as a measure of accuracy of our method are $E(M)=\left|M_{\infty}-M_{\infty}^{c}\right|, E\left(\theta_{\infty}\right)=\left|\theta_{\infty}-\theta_{\infty}^{c}\right|, E\left(\theta_{B}\right)=$ $\max \left|\theta_{B}\left(s_{i}\right)-\theta_{B}^{c}\left(s_{i}\right)\right|, y$ gap $=|y(s=0)-y(s=1)|$, and $x$ gap $=|x(s=0)-x(s=1)|$.
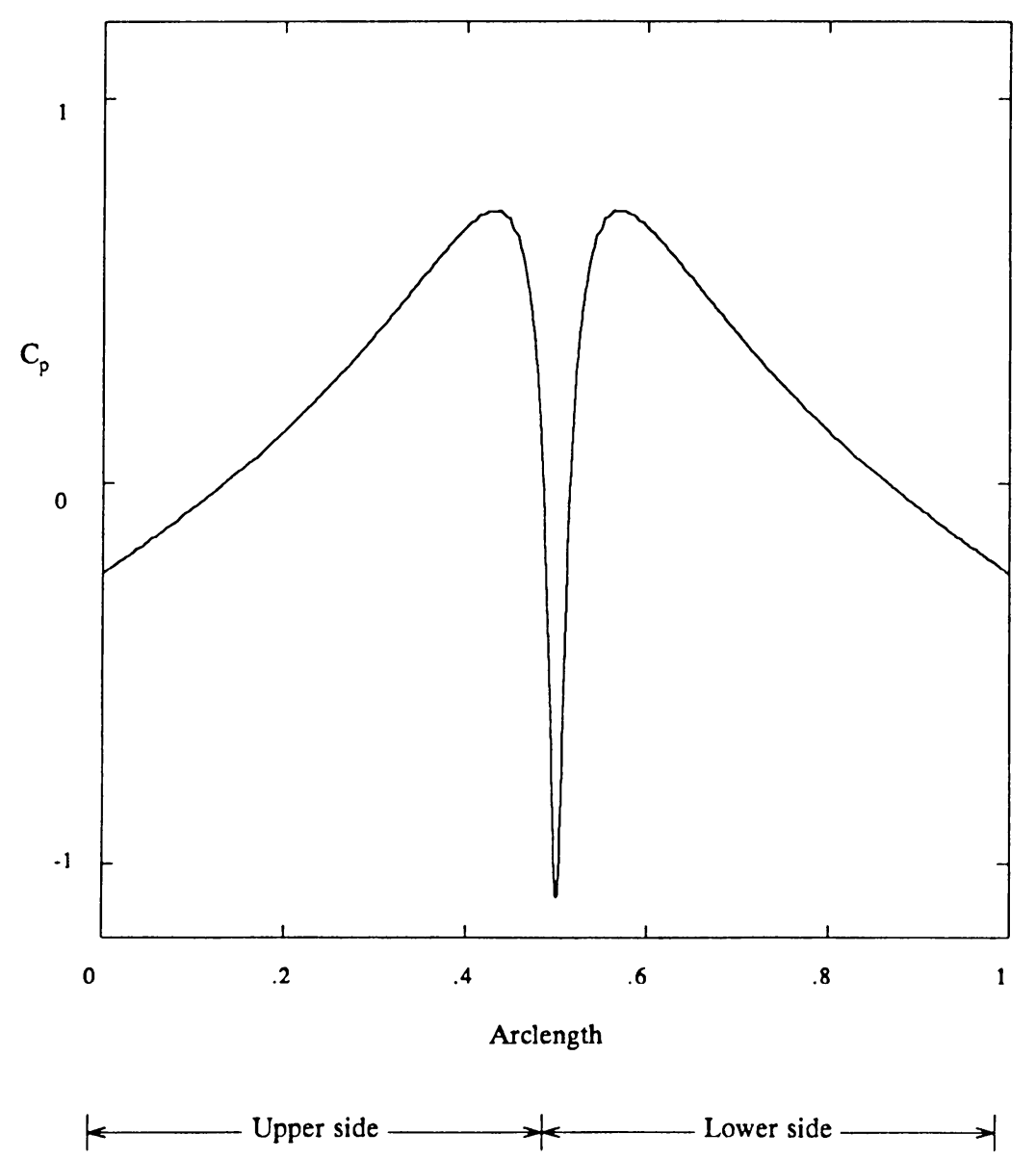

FIG. 2. Pressure distribution over a $12 \%$ thick Kutta airfoil from Euler solution (flo52s) at $M_{\infty}=0.6$ and $\alpha=0$. 
$x$ gap and $y$ gap (normalized by chord length) measure the gap at the trailing edge of the airfoil and $\theta_{B}$ refers to the body angle. The above diagnostics are a measure of numerical sources of error in our method and should ideally be zero.

We present a series of results. Fig. 2 shows the Euler pressure distribution over a $12 \%$ thick Kutta airfoil at free stream Mach number $M_{\infty}=0.6$ and $\theta_{\infty}=0$. The application of the above method then generates the body and also gives the computed values of the free stream Mach number, $M_{\infty}^{c}$, and the angle of attack, $\theta_{\infty}^{c}$. The number of iterations (see Sec. 4.1) required to converge to the solution using the linear elliptic solver was only six. Fig. 3 compares the designed airfoil with the original airfoil. We find that the agreement is excellent. Our method gives the free stream Mach number $M_{\infty}^{c}=0.60033$ and the angle of attack $\theta_{\infty}^{c}=0.00174$ radian (0.1 degree). We get the following values of our error diagnostics: $E(M)=0.00033$, $E\left(\theta_{\infty}\right)=0.00174, E\left(\theta_{B}\right)=0.00672, x$ gap $=0.00000$, and $y$ gap $=0.00133$. Notice that the designed airfoil is not closed and the gap is within about $1 \%$ of the airfoil thickness. In general, closure cannot be expected unless explicitly imposed either by some iterative or exact method. (We will report on the design of a closed airfoil in the near future.) The source of these errors is numerical as has been discussed before.

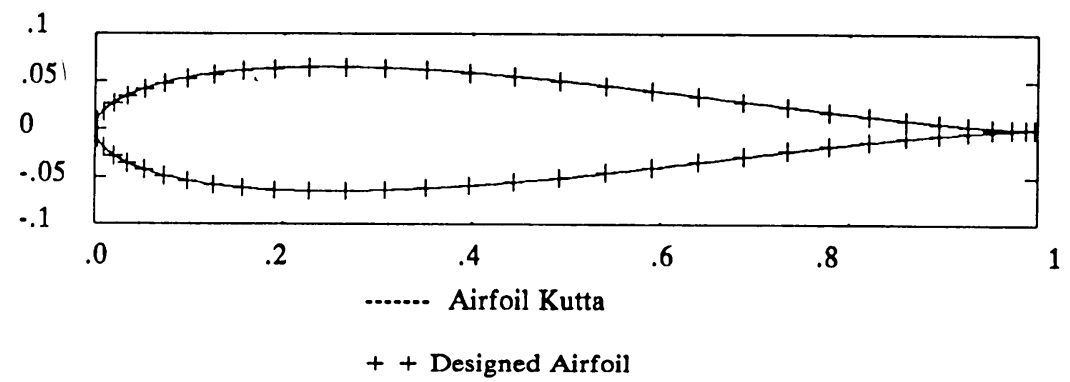

FIG. 3. Comparison of the $12 \%$ thick Kutta airfoil and the airfoil designed from the Euler pressure distribution of Fig. 2.

A more important quantity is the body angle which is likely to suffer maximum error near the leading edge since the body angle is a rapidly varying function of arclength there. Fig. 4 compares the body angle of the original airfoil with that of the designed airfoil as a function of arclength, and Fig. 5 compares the same in the leading edge region. Again notice that the agreement is excellent. The error in the body angle is $O\left(10^{-4}\right)$ everywhere except near the leading edge where the error is maximum and is given by $E\left(\theta_{B}\right)=0.00672$ radian.

Next we show the results based on the same airfoil but with an angle of attack. Fig. 6 shows the pressure distribution at $M_{\infty}=0.5$ and angle of attack $=2.0$ degrees. The number of iterations required to design by our method was five. In Fig. 7 we compare the designed airfoil with the exact airfoil and find that the agreement is again excellent. We find the computed free stream Mach number $M_{\infty}^{c}=0.50005$ and the angle of attack $\theta_{\infty}^{c}=2.05$ degrees. The values of the error diagnostics in this case are $E(M)=0.00005, E\left(\theta_{\infty}\right)=0.00087, E\left(\theta_{B}\right)=0.009, x$ gap $=0.00043$, and 


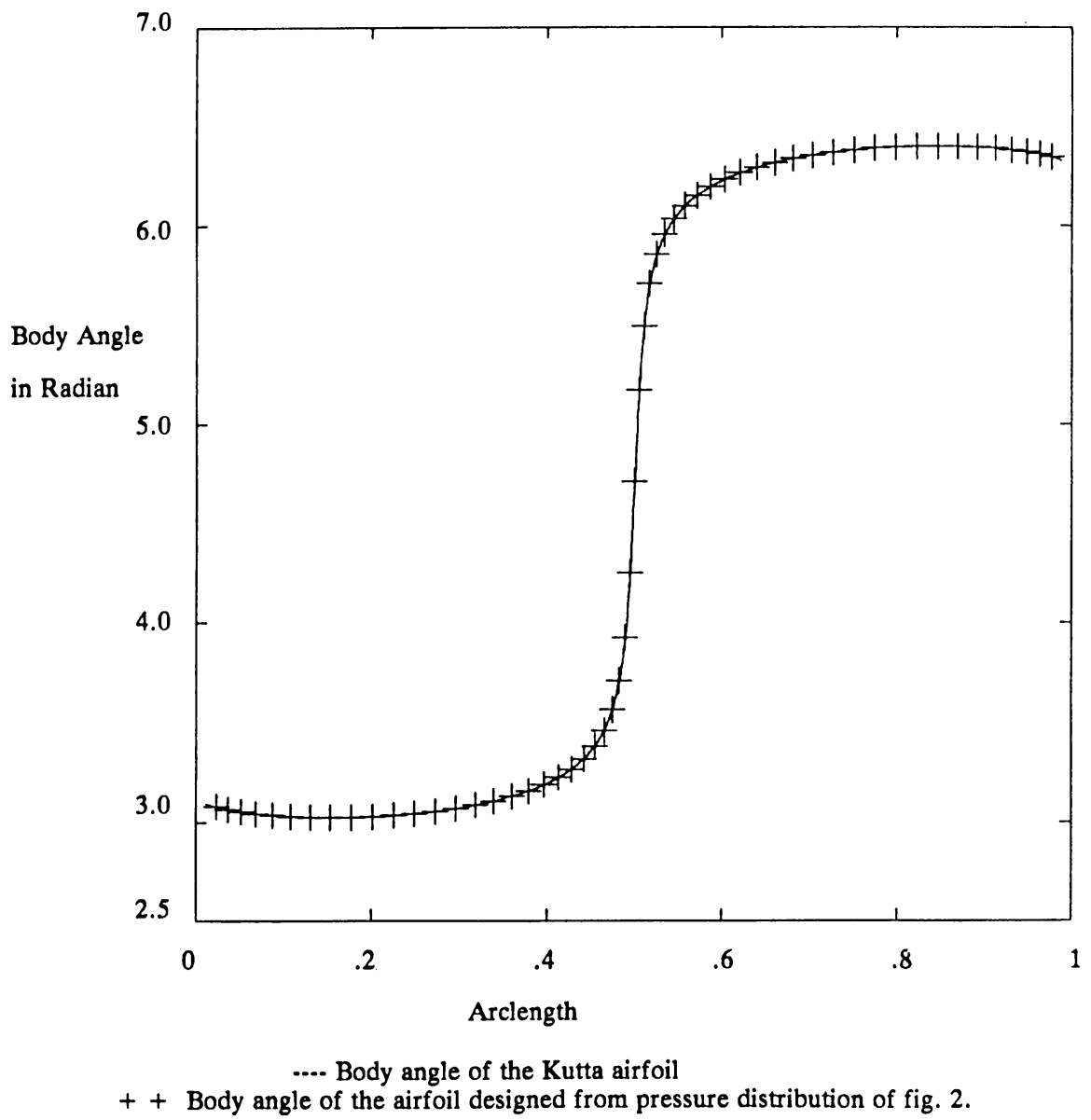

FIG. 4. Comparison of the body angle of the designed airfoil and of the Kutta airfoil as a function of arclength of the airfoil. The input pressure distribution of the designed airfoil is shown in Fig. 2.

$y$ gap $=0.00010$. The gap at the trailing edge is within $0.01 \%$ of the thickness of the airfoil which is negligibly small. Fig. 8 compares the computed and exact values of body angle as a function of arclength. Fig. 9 compares the same in the leading edge region. Here again we find the error in the body angle is $O\left(10^{-4}\right)$ everywhere except near the leading edge where it is maximum and is given by $E\left(\theta_{B}\right)=0.009$.

5. Conclusions. A new method for solving the inverse problem has been presented. The key point is that knowing the pressure distribution, the body is known in the potential plane. We find the body in the physical plane by solving one Dirichlet-type nonlinear boundary value problem. It is worth noting that, from a mathematical point of view, this problem is similar to the analysis (direct) problem, except that in the latter case one usually solves a Neumann-type problem. For computational simplicity, the potential plane was mapped into the interior of the unit circle and the problem was solved there. 


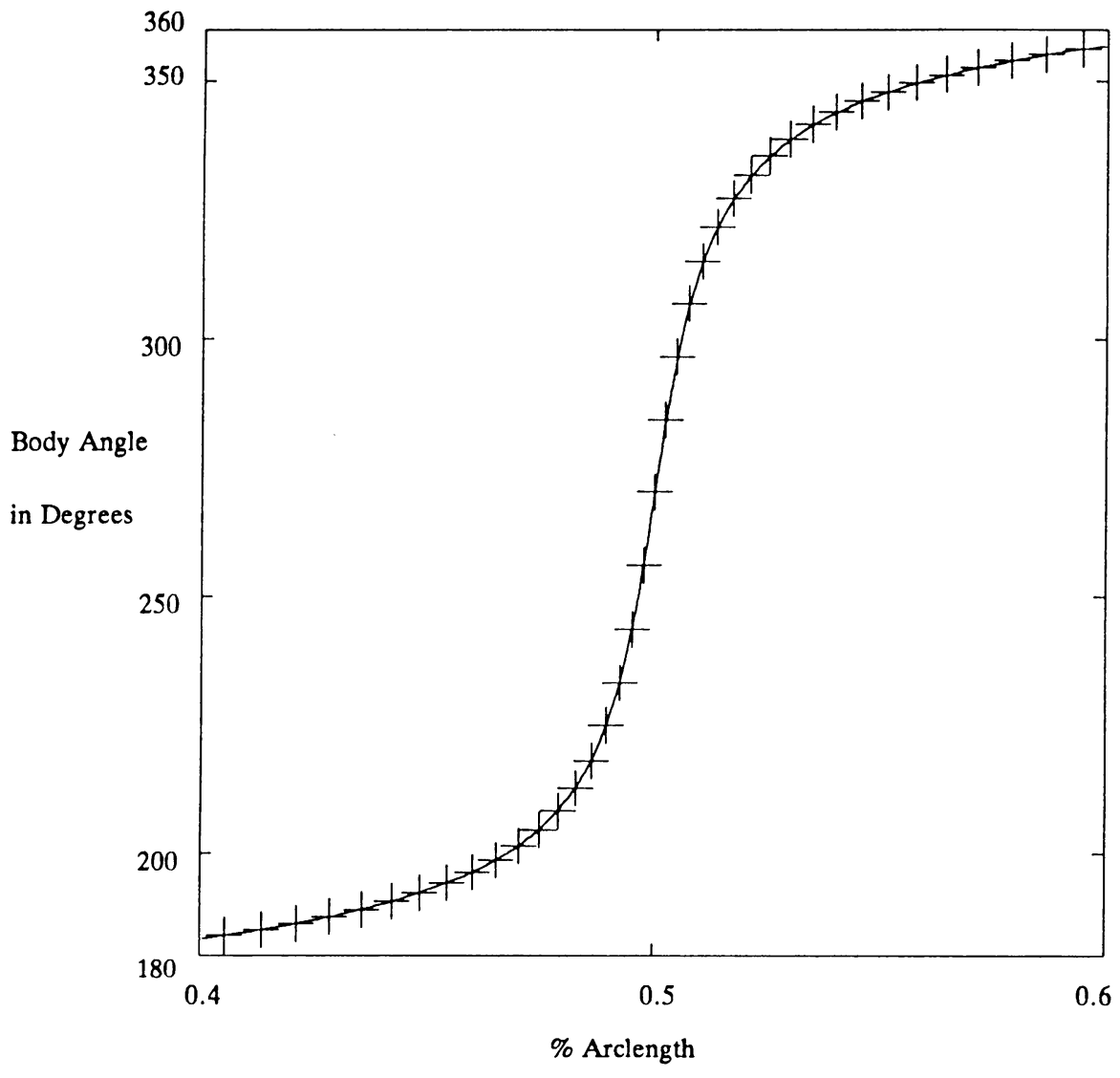

...... Body angle of the Kutta airfoil in the leading edge region

++ Body angle of the designed airfoil in the leading edge region

Fig. 5. Comparison of the body angle of the designed airfoil and of the Kutta airfoil as a function of arclength of the airfoil in the leading edge region. The input pressure distribution of the designed airfoil is shown in Fig. 2.

The solution to the inverse problem exists only if the computed free stream Mach number is the same as the Mach number corresponding to the input pressure distribution. In our formulation, the free stream Mach number is determined by the solution and thus the generation of an airfoil is always guaranteed. The computed Mach number then determines whether the input pressure distribution has a solution or not. In either case we can compute the pressure distribution on the designed airfoil at any Mach number we wish. This is an important feature of our method.

The method presented here is based on a mathematically exact formulation, and the only error is from numerical approximation. The numerical errors introduce a gap at the trailing edge. We are developing a closure procedure to obtain a specified gap (zero gap for closed airfoil) at the trailing edge. An obvious approach would be to use our method in an iterative mode. 

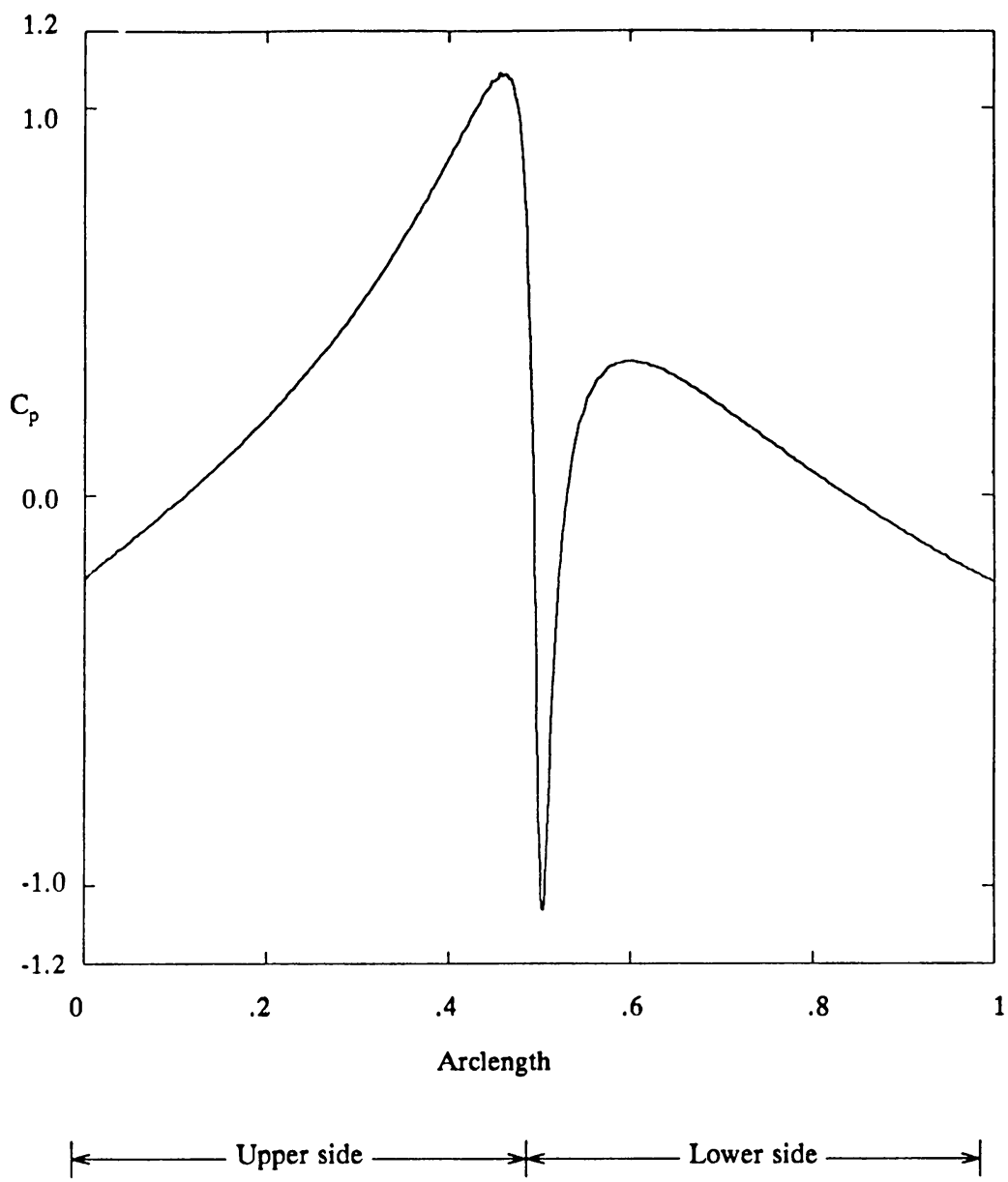

Fig. 6. Pressure distribution over a $12 \%$ thick Kutta airfoil from

Euler solution (flo52s) at $M_{\infty}=0.5$ and $\alpha=2$ degrees.

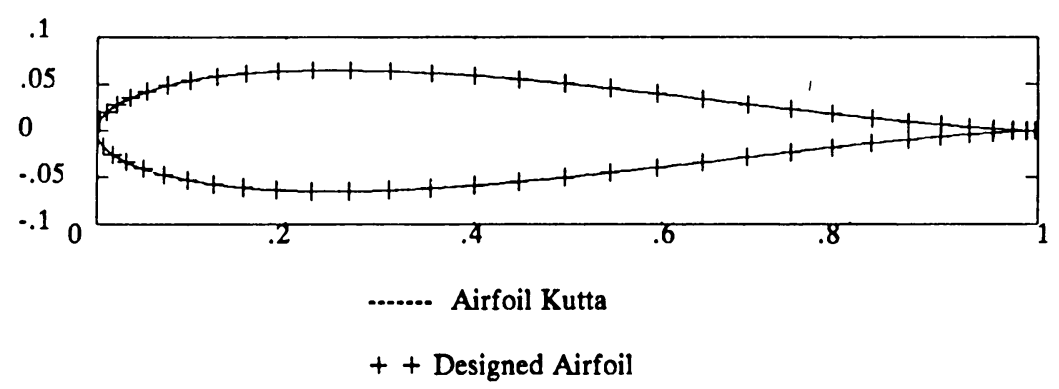

FIG. 7. Comparison of the $12 \%$ thick Kutta airfoil and the airfoil designed from Euler pressure distribution of Fig. 6.

We hope to extend this method to design supercritical airfoils using the ideas of fictitious gas (Fung et al [9], Yu [40], Sobieczky [29], Sobieczky [30] and references 


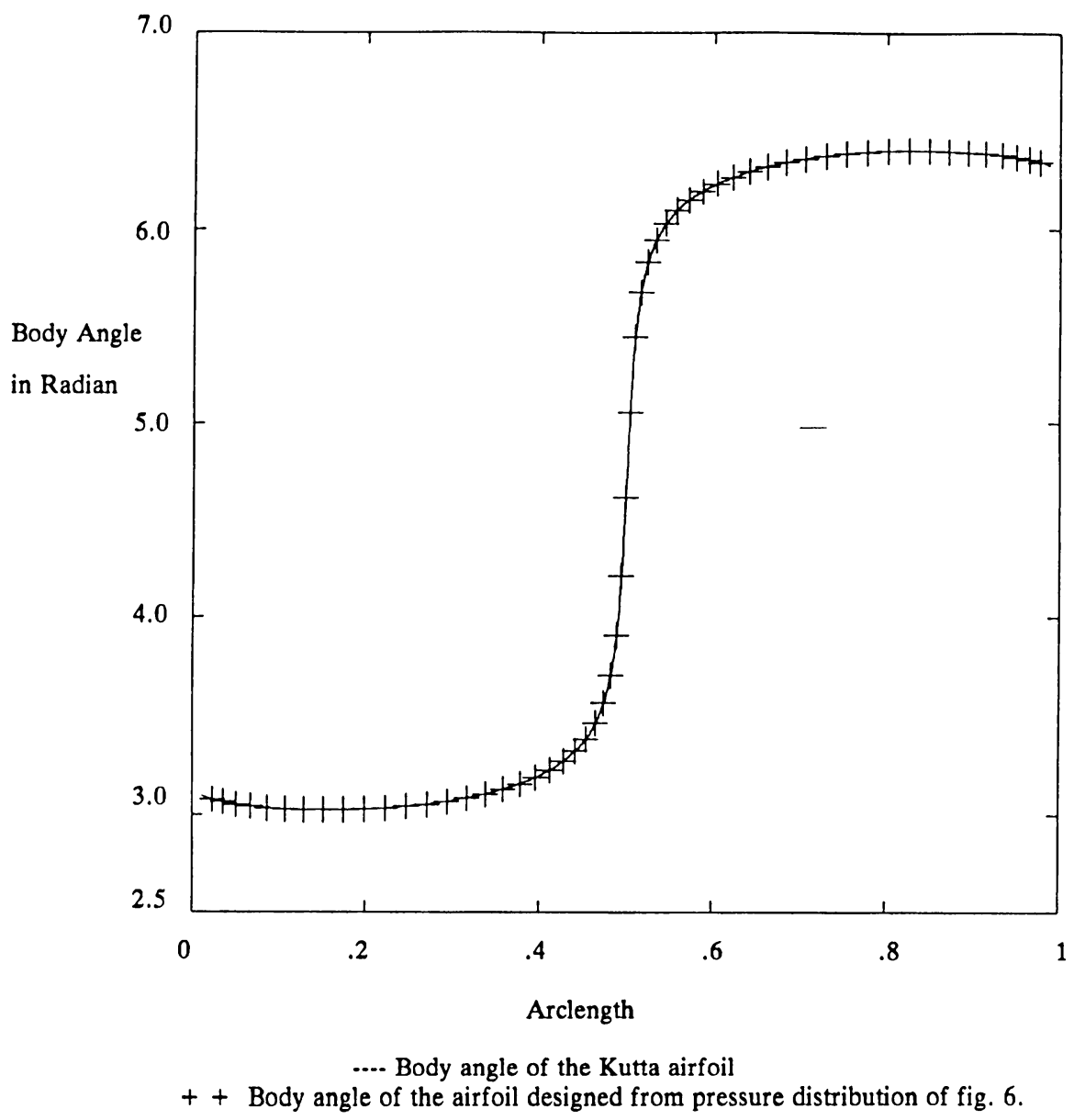

Fig. 8. Comparison of the body angle of the designed airfoil and of the Kutta airfoil as a function of arclength of the airfoil. The input pressure distribution of the designed airfoil is shown in Fig. 6.

therein, Sobieczky et al [31] for the supersonic region. Also, this method can be extended to design supercritical airfoils without making any use of fictitious gas.

The author thanks his thesis advisor Professor Lawrence Sirovich for helpful discussions. I also thank Professor James Glimm and Professor Paul Garabedian for constructive comments. I am indebted to Professor Marsha Berger for interesting discussions and for her patient reading and correcting of the manuscript. This work was supported by the National Aeronautics and Space Administration under Grant NSG-1617 and the Air Force Office of Scientific Research under Grant AFOSR-830336 at Brown University and by the Applied Mathematical Sciences subprogram of the Office of Energy Research, U. S. Department of Energy, under Contract No. DE-AC02-76ER03007 at New York University. This work was initiated at Brown University and extends results contained in my Ph.D. thesis (Daripa [5]). 


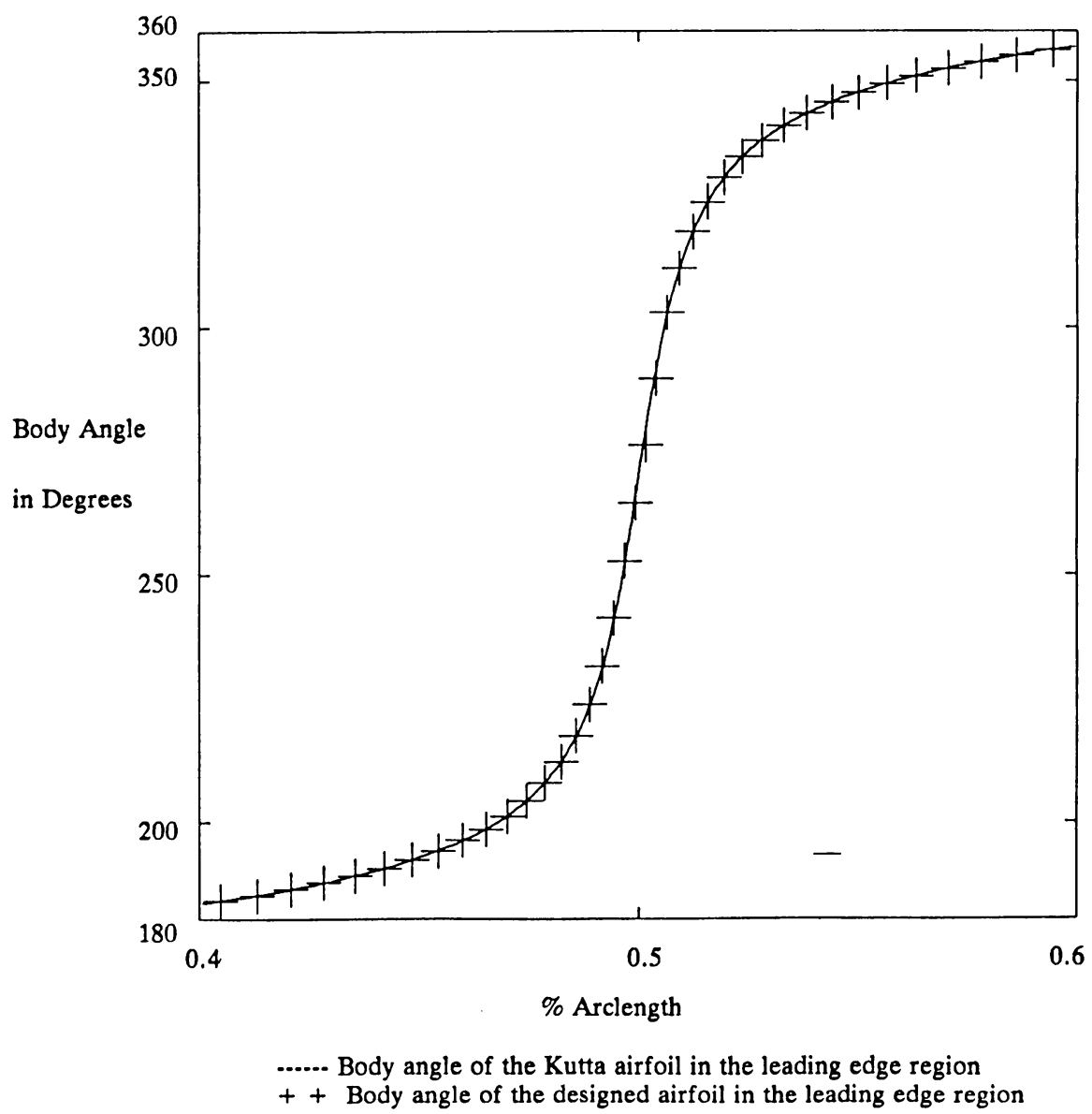

FIG. 9. Comparison of the body angle of the designed airfoil and of the Kutta airfoil as a function of arclength of the airfoil in the leading edge region. The input pressure distribution of the designed airfoil is shown in Fig. 6.

Appendix A. Derivation of Equations (42) and (51). We briefly derive the expressions for $\theta_{\alpha}$ and $\theta_{r} . \theta_{\alpha}$ can be written as

$$
\theta_{\alpha}=\theta_{\phi} \phi_{\alpha}+\theta_{\psi} \psi_{\alpha}
$$

From (1a,b) and the mapping function given by (15), we obtain

$$
\begin{aligned}
& \theta_{\phi}=K^{-1} D^{-1}\left(\nu_{\alpha} \psi_{\alpha}-\nu_{l} \phi_{\alpha}\right), \\
& \theta_{\psi}=-K D^{-1}\left(\nu_{\alpha} \phi_{\alpha}+\nu_{l} \psi_{\alpha}\right) .
\end{aligned}
$$

$D$ and $\nu_{l}$ in Eqs. (A2) and (A3) are given by

$$
D=\phi_{\alpha}^{2}+\psi_{\alpha}^{2} ; \quad \nu_{l}=\gamma \nu_{\gamma}
$$

Direct substitution of (A2) and (A3) into (A1) results in

$$
\theta_{\alpha}=D^{-1}\left\{\left(K^{-1}-K\right) \nu_{\alpha} \phi_{\alpha} \psi_{\alpha}-\left(K^{-1} \phi_{\alpha}^{2}+K \psi_{\alpha}^{2}\right) \nu_{l}\right\}
$$


On the unit circle $r=1$,

$$
\psi_{\alpha}=0, \quad D=\phi_{\alpha}^{2}
$$

which reduces $(\mathrm{A} 4)$ to

$$
\theta_{\alpha}=-K^{-1} \nu_{r} \quad \text { on } r=1 .
$$

$\theta_{r}$ can be written as

$$
\theta_{r}=\theta_{\phi} \phi_{r}+\theta_{\psi} \psi_{r}
$$

Direct substitution of (A2) and (A3) into (A6) and using the fact the mapping (15) is conformal, we obtain

$$
\theta_{r}=D^{-1} r^{-1}\left\{\left(K^{-1} \psi_{\alpha}^{2}+K \phi_{\alpha}^{2}\right) \nu_{\alpha}-\left(K^{-1}-K\right) \nu_{l} \phi_{\alpha} \psi_{\alpha}\right\} .
$$

It can be easily seen from (15) that

$$
\psi_{\alpha}=0, \quad \text { on } \alpha=\alpha_{*}=\frac{\pi}{2}-\alpha_{0} \text { and } 3 \frac{\pi}{2}-\alpha_{0} .
$$

Then (A7) reduces to

$$
\theta_{r}=\left.\frac{K(M)}{r} \nu_{\alpha}(r)\right|_{\alpha=\alpha_{*}} .
$$

Appendix B. Stagnation point estimate of right-hand side of Equation (37). In the following we determine the nature of singularity of the right-hand side of Eq. (37) at a stagnation point. From (53) and (60),

$$
M= \begin{cases}O\left(\alpha^{\delta}\right) & \text { as } \alpha \rightarrow 0, \\ O\left(\left|\alpha-\alpha_{s}\right|\right) & \text { as } \alpha \rightarrow \alpha_{s} .\end{cases}
$$

From (14) and (B1),

$$
f(M)= \begin{cases}O\left(\alpha^{4 \delta}\right) & \text { as } \alpha \rightarrow 0, \\ O\left(\left|\alpha-\alpha_{s}\right|^{4}\right) & \text { as } \alpha \rightarrow \alpha_{s} .\end{cases}
$$

We recall from Sec. 3.3.1 that $\delta$ above refers to $\theta_{t} / \pi$ where $\theta_{t}$ is the trailing edge angle. $K(M)$ (see Eq. (4)) can be expanded in $M$ to give

$$
K=R\left\{1-\frac{\gamma+1}{8} M^{4}+O\left(M^{6}\right)\right\}
$$

where

$$
R=c\left\{\frac{2}{\gamma+1}\right\}^{\frac{1}{\gamma-1}}
$$

From (B1) and (B3),

$$
\begin{aligned}
1-K^{2} & =1-R^{2}+R \frac{(\gamma+1)}{4} M^{4}+O\left(M^{6}\right) \\
& =\left(1-R^{2}\right)+ \begin{cases}O\left(\alpha^{4 \delta}\right) & \text { as } \alpha \rightarrow 0, \\
O\left(\left|\alpha-\alpha_{s}\right|^{4}\right) & \text { as } \alpha \rightarrow a_{s} .\end{cases}
\end{aligned}
$$


A simple calculation using the mapping function (15) gives the following estimates as $\alpha \rightarrow 0$ for $\theta_{t}>0$ :

$$
\begin{aligned}
& D L_{\phi \phi}= \begin{cases}O\left(\frac{1}{\alpha^{2}}\right) & \text { as } \alpha \rightarrow 0, \\
O\left(\frac{1}{\left(\alpha-\alpha_{s}\right)^{2}}\right) & \text { as } \alpha \rightarrow \alpha_{s}\end{cases} \\
& \sqrt{D L_{\phi}}= \begin{cases}O\left(\frac{1}{\alpha}\right) & \text { as } \alpha \rightarrow 0, \\
O\left(\frac{1}{\left|\alpha-\alpha_{s}\right|}\right) & \text { as } \alpha \rightarrow \alpha_{s},\end{cases} \\
& \sqrt{D L_{\psi}}=O(1) \text { as } \alpha \rightarrow 0 \text { or } \alpha_{s} .
\end{aligned}
$$

From (B5) and (B8) we obtain the following estimates for the right-hand side (RHS) of Eq. (37):

$$
\mathrm{RHS}= \begin{cases}O\left(\alpha^{4 \delta-2}\right) & \text { as } \alpha \rightarrow 0, \\ O\left(\left(\alpha-\alpha_{s}\right)^{2}\right) & \text { as } \alpha \rightarrow \alpha_{s} .\end{cases}
$$

In arriving at the above estimate, the value of $R$ in equation (B5) has been taken to be one, which determines the value of the constant $c$ from (B4). The constant $c$ is then given by

$$
c=\left\{\frac{\gamma+1}{2}\right\}^{\frac{1}{\gamma-1}}
$$

From (B9) we see that the RHS is regular at the front stagnation point. The RHS will be singular at the front stagnation point if $c$ is taken to be different from that given by (B10). However, it is singular at the trailing edge if $0<\theta_{t}<\pi / 2$ and regular for $\pi / 2 \leq \theta_{t} \leq 2 \pi$ and $\theta_{t}=0$. For $\theta_{t}=0$, estimates in (B2), (B6) to (B8) are of $O(1)$ as $\alpha \rightarrow 0$ and hence the RHS remains regular.

\section{REFERENCES}

[1] A. B. Arlinger, An exact method of two-dimensional airfoil design, SAAB Tech. Notes TN 67 (1970)

[2] L. Bers, Mathematical Aspects of Subsonic and Transonic Gas Dynamics, John Wiley and Sons, New York, 1958

[3] F. Bauer, P. R. Garabedian, and D. Korn, Supercritical wing sections III, Lecture Notes in Economics and Mathematical Systems 150, Springer-Verlag, New York, 1977

[4] L. A. Carlson, The inverse transonic flow calculations using experimental pressure distributions, AIAA J. 12 (4), 571-572 (1976)

[5] P. Daripa, The Direct and Inverse Problems in Gas Dynamics, Ph.D. Thesis, Brown University, Providence, R.I., 1985

[6] P. Daripa and L. Sirovich, Exact and approximate gas dynamics using the tangent gas, J. Comput. Phys. 62, 400-413 (1986)

[7] P. Daripa and L. Sirovich, An inverse method for subcritical flows, J. Comput. Phys. 63, 311-328 (1986)

[8] W. H. Davis, Technique for developing design tools from the analysis methods of computational aerodynamics, AIAA Pap. 79-1529, 1979

[9] K.-Y. Fung, H. Sobieczky, and A. R. Seebass, Shock-free wing design, AIAA J. 18, 1153-1158 (1980)

[10] P. R. Garabedian, Design methods for supercritical airfoils, Proceedings on ICIDES, University of Texas, Austin, 133-143 (1984) 
[11] P. R. Garabedian and D. G. Korn, Numerical design of transonic airfoils, In Numerical Solution of Partial Differential Equations. II, ed. Bert Hubbard, Academic Press, New York, 1971, p. 253

[12] P. R. Garabedian and G. McFadden, Computational fluid dynamics of airfoils and wings, Transonic, shock and multidimensional flows: advances in scientific computing, ed. by R. E. Meyer, Academic Press, New York, 1982, pp. 1-16

[13] A. H. Glauert, Aerofoil and Airscrew Theory, Cambridge University Press, 1926

[14] S. Goldstein, A Theory of Aerofoils of Small Thickness, Rep. ARC. 6225, London, 1942

[15] N. D. Halsey, Methods for the design and analysis of jet-flapped airfoils, AIAA Pap. 74-188, 1974

[16] A. A. Hassan, A method for the design of shock-free slender bodies of revolution, AIAA J. 24 (5) (1986)

[17] A. A. Hassan, H. Sobieczky, and A. R. Seebass, Subcritical and supercritical airfoils for a given pressure distribution, AIAA Pap. 81-1233, 1981

[18] A. A. Hassan, H. Sobieczky, and A. R. Seebass, Subsonic airfoils with a given pressure distribution, AIAA J. 22 (9) (1984)

[19] R. Hicks and G. Vanderplaats, Application of numerical optimization to the design of supercritical airfoils without drag creep, Society of Automotive Engineers Pap. 770440, 1977

[20] R. Hicks, G. M. Vanderplaats, E. M. Murman and R. R. King, Airfoil section drag reduction at transonic speeds by numerical optimization, NASA TMX. 73097, 1976

[21] F. T. Johnson, A general panel method for the analysis and design of arbitrary configurations in incompressible flows, NASA CR. 3079, 1980

[22] F. T. Johnson and P. E. Rubbert, Advanced panel-type influence coefficient methods applied to subsonic flows, AIAA Pap. 75-50, 1975

[23] M. J. Langley, Numerical methods for two-dimensional and axisymmetric flows, ARA memo. 143, 1973

[24] H. W. Liepmann and A. Roshko, Elements of Gas Dynamics, John Wiley and Sons, Inc., 1957

[25] M. J. Lighthill, A new method of two-dimensional aerodynamic design, ARC, R\&M 2112, 1945

[26] G. B. McFadden, An artificial viscosity method for the design of supercritical airfoils, Ph.D. Thesis, N.Y. University, New York, N.Y., 1979

[27] J. Sanz, $A$ well posed boundary value problem in transonic gas dynamics, Comm. Pure Appl. Math. 31, 671-679 (1978)

[28] J. W. Sloof, A survey of computational methods for subsonic and transonic aerodynamics design, Proceedings on ICIDES, University of Texas, Austin, 1-68 (1984)

[29] H. Sobieczky, Related analytical, analog and numerical methods in transonic airfoil design, AIAA Pap. 79-1556, 1979

[30] H. Sobieczky, Analytical tools for systematic transonic design, Proceedings of ICIDES, University of Texas, Austin, 85-112 (1984)

[31] H. Sobieczky and A. R. Seebass, Supercritical airfoil and wing design, Ann. Rev. Fluid Mech. 16, 337 (1984)

[32] T. Strand, Exact method of designing airfoils with given velocity distribution in incompressible flow, J. Aircraft 10, 651-659 (1973)

[33] B. S. Stratford, Rep. \& Mem. Aero. Res. Coun. 3002, London, 1954

[34] B. S. Stratford, The prediction of separation of the turbulent boundary layers, J. Fluid Mech. 5, 1-16 (1959)

[35] B. S. Stratford, An experimental flow with zero skin friction throughout its region of pressure rise, J. Fluid Mech. 5, 17-35 (1959)

[36] P. N. Swarztrauber and R. A. Sweet, Efficient Fortran subprograms for the solution of elliptic equations, NCAR TN/IA 109, 138 (1975)

[37] T. L. Tranen, A rapid computer aided transonic airfoil design method, AIAA Pap. 74-501, 1974

[38] G. Volpe, The inverse design of closed airfoils in transonic flow, AIAA Pap. 83-0504, 1983

[39] G. Volpe and R. E. Melnik, The role of constraints in the inverse design problem for transonic airfoils, AIAA Pap. 81-1233, 1981

[40] N. J. Yu, Efficient transonic shock-free wing redesign procedure using a fictitious gas method, AIAA J. 18, 143 (1980) 